\title{
PROCESO ATENCIÓN DE ENFERMERÍA APLICADO A USUARIO CON HEMORRAGIA SUBARACNOIDEA POSTRAUMÁTICA USANDO EL MODELO AREA
}

\author{
NURSING PROCESS APPLIED TO \\ USER WITH POSTUMATIC SUBARACNOIDAL \\ HEMORRHAGE USING THE AREA MODEL
}

Recibido: 30 marzo 2017 Aceptado: 30 noviembre 2017

Correspondencia: Avenida aeropuerto $\mathrm{Mz} 23 \mathrm{~h}$ L† 2 Estado de México. México Correo electrónico: in-u-13@hotmail.com

\section{Autores:} \\ E.L.E. Gómez-Juárez S. \\ Estudiante de pregrado de la Licenciatura en \\ enfermería Facultad de Estudios Superiores Zaragoza. \\ Universidad Nacional Autónoma de México Ciudad \\ de México. México *Autor para correspondencia: \\ Correo electrónico: in-u-73@hotmail.com \\ (S. Gómez-Juárez). \\ L.E.O. Rosales-Ortega J. \\ Profesor de asignatura, Facultad de Estudios Superiores \\ Zaragoza. Universidad Nacional Autónoma de México \\ Ciudad de México. México*:Correo electrónico: \\ enfjaro2@hotmail.com
}

Palabras clave: proceso atención de enfermería; hemorragia subaracnoidea; modelo AREA.

Key words: nursing care process; subarachnoid hemorrhage; model AREA 
RESUMEN

Se eligió un usuario con quien se aplicó la metodología del Proceso de Atención de Enfermería (PAE), empleando la taxonomía North American Nursing Diagnosis Association (NANDA), Nursing Outcomes Classification (NOC), Nursing Interventions Classification (NIC), Se valoró al usuario a través de un instrumento basado en la teoría de Dorothea Orem, identificando los requisitos de autocuidado universales, del desarrollo y de desviación a la salud; el usuario se encontraba hospitalizado en el servicio de Unidad de Cuidados Intensivos con un Diagnostico de Hemorragia Subaracnoidea, durante el periodo postquirúrgico tardío. Se llevó a cabo un razonamiento diagnóstico de enfermería mediante el Modelo de Análisis de Resultado del Estado Actual (AREA), cabe destacar que se implementa el lenguaje sugerido por la NANDA y el uso de un diagnóstico de riesgo como principal problema, haciendo énfasis en las aclaraciones de la misma en su nueva edición 2015-2017, para después planificar e implementar los cuidados de enfermería, los cuales fueron evaluados mediante la escala Diana, de manera cualitativa porcentual, logrando tener un avance del $90 \%$ en la evaluación final en el resultado esperado principal: perfusión tisular: cerebral.

Palabras clave: proceso atención de enfermería; hemorragia subaracnoidea; modelo AREA.

\section{ABSTRACT}

A user with whom the Nursing Care Process (PAE) methodology was used, using the North American Nursing Diagnosis Association (NANDA), Nursing Interventions Classification (NIC), Nursing Outcomes Classification (NOC) Through an evaluation instrument based on the theory of Dorothea Orem, identifying the universal requirements of auto care, of the development, and of diversion to the health; the user was hospitalized in the service of Unit of Intensive care by Diagnosis of Hemorrhage subaracnoidea, during the postsurgical late period. A diagnostic reasoning of infirmary was carried out by means of the Model of Analysis of Result of the Current State (AREA), it is necessary to emphasize that the suggested language is implemented by the NANDA and the use of a diagnosis of risk as principal problem, doing emphasis in the explanations of the same one in his new edition 2015-2017, later to plan and to help taken care of infirmary, which were evaluated by means of the Diana scale, in a qualitative perceptual way, managing to have A $90 \%$ of improvement in the final evaluation in the main expected outcome: tissue perfusion: cerebral.

Key words: nursing care process; subarachnoid hemorrhage; model AREA 


\section{INTRODUCCIÓN}

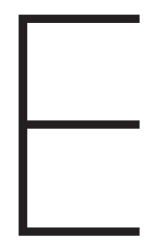

I Proceso de Atención de Enfermería (PAE), es un método sistemático y organizado para brindar cuidado individualizado y holístico, es el método que aplica la enfermera para identificar necesidades y problemas del paciente, con una base dialéctica, cognoscitiva y educativa, incorporando la taxonomía North American Nursing Diagnosis Association (NANDA), Nursing Outcomes Classification (NOC) y por último la incorporación de la clasificación intervenciones Nursing Interventions Classification (NIC). ${ }^{1-4}$

El Modelo de Análisis de Resultado del Estado Actual (AREA), establece una estructura para el razonamiento clínico que incluye los problemas centrándolos en los resultados esperados en la práctica enfermera ${ }^{5}$. El modelo AREA plantea una alternativa al razonamiento clínico tradicional. Muchos diagnósticos NANDA se relacionan entre sí y pueden representarse de forma gráfica a través de lo que se denomina red lógica, en la cual se identifican los diagnósticos enfermeros principales y las complicaciones potenciales en los terrenos interdependiente y dependiente. La manera de abordar estas relaciones, pretende que con las intervenciones que la enfermera realice del diagnóstico principal, los diagnósticos secundarios se resuelvan de manera que el usuario regrese a un estado óptimo de salud. ${ }^{6,7}$

Los diagnósticos de riesgo pueden ser realmente los de máxima prioridad en un paciente, el uso previo del término diagnóstico real puede haber dado lugar a esta confusión; algunas personas interpretan esto como que el diagnostico real (enfocado en el problema) es más real que el de riesgo. Debido a que el terminorelacionado cons> se utiliza para sugerir una etiología en los diagnósticos enfocados en el problema, ya que sólo hay vulnerabilidad a un problema cuando se utiliza un diagnóstico de riesgo
NANDA-I ha decidido recomendar el uso de la frase como lo demuestra para referirse a la evidencia del riesgo que existe. ${ }^{2}$

La hemorragia subaracnoidea (HSA) se define como el escape de sangre a partir de un vaso arterial o venoso hacia el espacio subaracnoideo, que en condiciones normales está ocupado sólo por líquido cefalorraquídeo, nervios y vasos craneales; puede ser de dos tipos: espontánea (ruptura de aneurismas vasculares intracraneales en 80\%) o traumática. ${ }^{8.9}$ La hemorragia subaracnoidea postraumática (HSAPT), es un hallazgo que se presenta con una frecuencia de 40\% según los datos del American Traumatic Coma Data Bank (TCDB). La HSA traumática es la forma más común y se produce por la laceración de las venas o arterias corticales que atraviesan el espacio subaracnoideo, pero también puede resultar de contusiones o laceraciones.

Por lo general, existe una elevada concentración de los productos de la sangre alrededor del origen de la hemorragia, pero la HSA originada de una fuente local tiende a ser difusa y llega a extenderse a los componentes del espacio subaracnoideo. La hemorragia también puede afectar a las estructuras del parénquima cerebral y el sistema ventricular adyacente. Al ocurrir la hemorragia se eleva la presión intracraneal (PIC) y, como consecuencia, disminuye de forma aguda la presión de perfusión cerebral. La hipoperfusión produce isquemia cerebral aguda y pérdida de la conciencia.

En la mayoría de las poblaciones la incidencia de la HSA se mantiene estable en aproximadamente 10 de cada 100000 habitantes/año (rango entre 2 y 20 según la población estudiada). Aunque a nivel internacional pueden existir variaciones entre las regiones, generalmente la incidencia reportada es alta en los Estados Unidos de Norteamérica, Europa

\section{5}

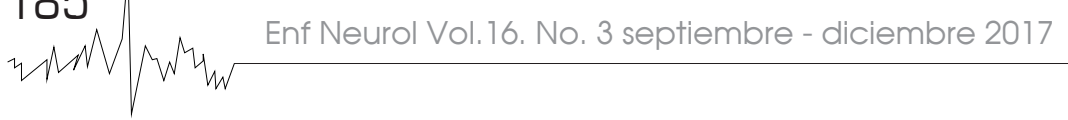


y Japón. Sin embargo, es baja en Nueva Zelanda, Qatar, China, India y Sudáfrica, probablemente por subestimación, dificultades en el acceso a los recursos diagnósticos y variaciones en los factores étnicos/genéticos. Este tipo de ictus predomina en la mujer de edad media, con una relación 3:2 y un pico de incidencia entre los 50 y 60 años de edad. Cuando aparece antes de la cuarta o después de la sexta década de la vida es más común en el sexo masculino. ${ }^{10}$

La HSA representa solamente el 2-5\% de los ictus, pero causa cerca del 25\% de los fallecimientos relacionados al ictus. Aproximadamente el 50-70\% de los pacientes con HSA fallece en los primeros 30 días. El 20-25\% fallece antes de llegar al hospital y en los pacientes hospitalizados existe un promedio de $40 \%$ de mortalidad en el primer mes. El riesgo de muerte súbita es superior en los aneurismas de la circulación posterior. Entre el 20-30\% de los sobrevivientes quedan con secuelas neurológicas incapacitantes. Los estudios de calidad de vida sugieren que menos de un tercio de los enfermos recuperan su ocupación y estilo de vida previo a los 18 meses. $^{10}$

El cuadro clínico es muy variable, puede cursar el multidrogos resistentes e con deterioro del estado de conciencia, cefalalgia, nausea, vómito, así como datos de rigidez de nuca y datos de focalización neurológica. La clasificación clínica de Hunt y Hess es muyútil para estadificar la gravedad del padecimiento.

El tratamiento dependerá de la causa de la hemorragia y las complicaciones, una opción es el tratamiento quirúrgico por medio de craneotomía. ${ }^{11}$

\section{Caso clínico}

Masculino 78 años de edad, internado en el servicio de Unidad de Cuidados Intensivos Adultos, con 10 días de estancia, se encuentra en estado de sedación con midazolam (Ramsay 6) y Glasgow de 6 puntos, palidez generalizada de tegumentos, hermodinámicamente inestable con tendencia a la hipertensión e hipertermia, ( T/A: 142/ 65 temperatura: $38^{\circ} \mathrm{C}$ FR: 26 FC: 76) normocéfalo, posoperado de drenaje por craneotomía, herida quirúrgica temporal izquierda con bordes bien afrontados, con alopecia, apertura ocular espontánea, pupilas isocóricas, normoreflexicas, intubación orotraqueal, cánula de \#7 apoyo ventilatorio asisto control con parámetros ventilatorios FlO2 40. PEEP 4, l:E1:2:1 FREC 27 y con presencia de sonda nasogástrica, palidez de mucosas, cuello cilíndrico, no palpables adenomegalias, tórax en tonel, se auscultan campos pulmonares con presencia de estertores, ruidos cardiacos con buen tono e intensidad, PVC 14 cc, ruidos peristálticos 2 por minuto, no viceromegalias, miembros torácicos con catéter central largo permeable, miembros torácicos y pélvicos presentando anasarca evaluando con signo de Godet positivo de +++, genitales íntegros con sonda vesical \# 14, diuresis color ámbar, oligúrico.

En la segunda etapa del proceso se desprenden los diagnósticos de enfermería reales y de riesgo, conforme los requisitos alterados y los factores de riesgo que el paciente presenta, así como la realización de intervenciones de enfermería dirigidas a la pronta recuperación o a mantener su estado de salud-enfermedad en su estancia. Con base en las problemáticas encontradas y los factores de riesgo detectados, se implementaron cuidados con el fin de mantener el estado de salud-enfermedad para corregir y mejorar su estado. En la ejecución se realizaron acciones de enfermería para la mejoría de la paciente, como son: mejora de la perfusión tisular, manejo de líquidos, mejora nutricional administración de medicamentos, cuidados a sonda nasogástrica (SNG), monitorización hemodinámica, aspiración de secreciones por tubo endotraqueal-boca y control de líquidos, entre otros. En la evaluación se analizaron los datos sobre cómo el paciente respondió al tratamiento y los cuidados que se le brindaron.

Enf Neurol Vol. 16. No. 3 septiembre - diciembre 2017 Why 186 
Esquema 1. Razonamiento diagnóstico modelo AREA

Riesgo de perfusión tisular cerebral ineficaz como lo demuestra lesión cerebral (hemorragia subaracnoidea postraumática)

Deterioro de la ventilación espontánea r/c fatiga de músculos accesorios $\mathrm{m} / \mathrm{p}$ disminución de la cooperación, disminución de la saturación de oxígeno
Hipertermia $\mathrm{r} / \mathrm{c}$ traumatismo craneoencefálico $\mathrm{m} / \mathrm{p}$ calor al tacto temperatura corporal $\left(38^{\circ} \mathrm{C}\right)$
Exceso de volumen de líquidos $\mathrm{r} / \mathrm{c}$ compromiso de de los mecanismos reguladores $\mathrm{m} / \mathrm{p}$ anasarca, cambios de la presión arterial

Desequilibrio nutricional: ingesta inferior a las necesidades $\mathrm{r} / \mathrm{c}$ incapacidad para ingerir alimentos (estado de sedación) $\mathrm{m} / \mathrm{p}$ pérdida de peso, palidez de mucosa

Riesgo de deterioro de la integridad cutánea como lo demuestra inmovilización física, estado de desequilibrio nutricional

\section{Red Diagnóstica de Enfermería basada en modelo AREA}

\section{En la presente priorización se abordo un diagnóstico de riesgo como principal, debido a que al ocurrir la hemorragia se eleva la presión intracraneal (PIC), y como consecuencia disminuye de forma aguda la presión de perfusión cerebral}

Tabla 1. Plan de Cuidados de Enfermería; Riesgo de perfusión tisular cerebral ineficaz.

DIAGNÓSTICO DE ENFERMERIA: Riesgo de perfusión tisular cerebral ineficaz como lo demuestra lesión cerel (hemorragia subaracnoidea Post-traumática)

DOMINIO: 09: afrontamiento/ tolerancia al estrés

CLASE: 03: estrés neurocomportamental

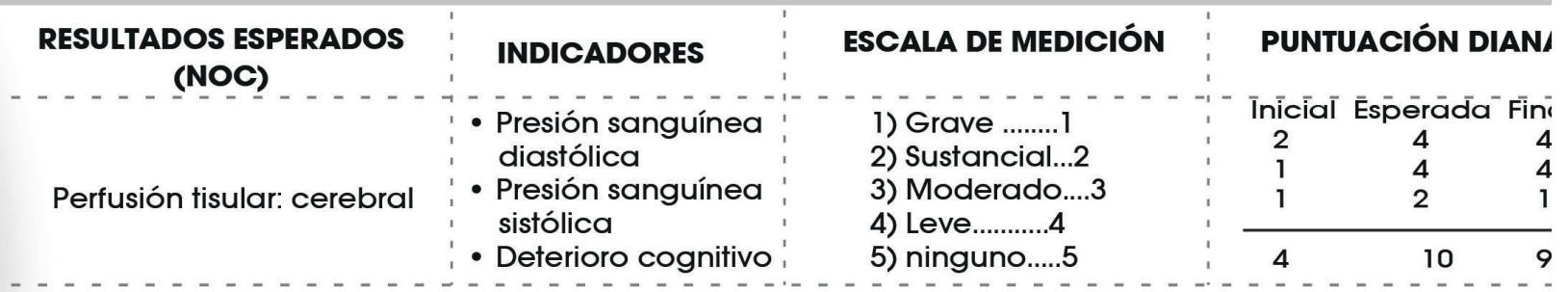

INTERVENCIÓN: Mejora de la perfusión cerebral

\section{ACTIVIDADES:}

- Monitorizar la presión arterial media

- Monitorizar la PVC

- Consultar con el médico para determinar los parámetros hemodinámicos y mantener dichos para metr dentro de ese rango.

- Evitar la flexión del cuello y la flexión extrema de la cadera/rodilla.

- Administrar y vigilar los efectos de los diuréticos osmóticos y de asa.

- Administrar anticoagulantes.

- Monitorizar la aparición de signos de hemorragia( búsqueda de sangre en heces y en drenaje nasogástriı

- Monitorizar estado neurológico 
Tabla 2. Plan de Cuidados de Enfermería; Deterioro de la ventilación espontánea.

DIAGNÓSTICO DE ENFERMERÍA: Deterioro de la ventilación espontanea $\mathrm{r} / \mathrm{c}$ fatiga de músculos accesorios $\mathrm{m} / \mathrm{p}$ disminución de la cooperación, disminución de la saturación de oxígeno DOMINIO:04: actividad/reposo

CLASE:04: respuestas cardiovasculares/pulmonares

\section{RESULTADOS ESPERADOS} (NOC)

Respuesta de la ventilación mecánica: adulto

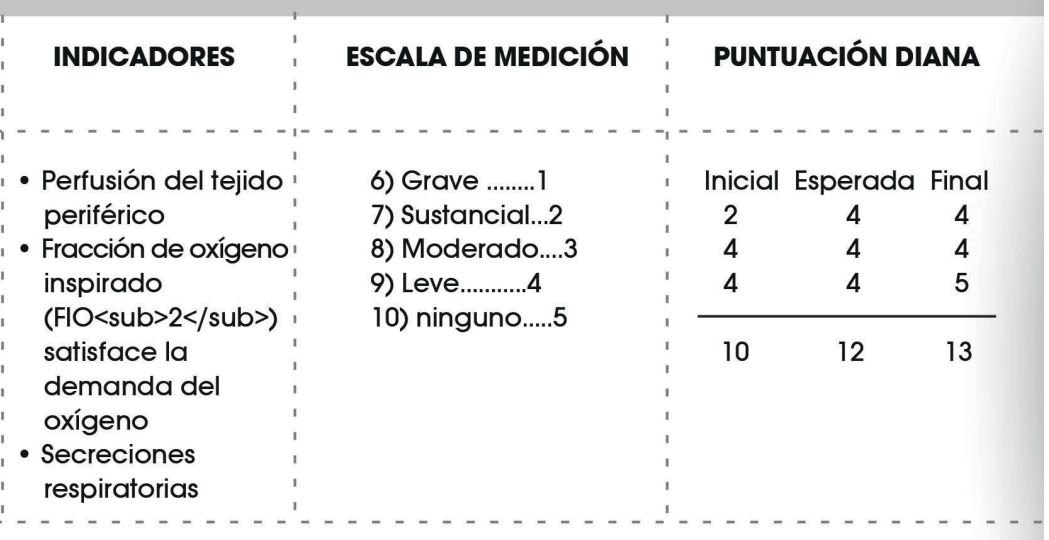

INTERVENCIÓN: Manejo de las vías aéreas artificiales ACTIVIDADES:

- Emplear precauciones universales

- Realizar aspiración endotraqueal.

- Cambiar las cintas/sujeción del tubo endotraqueal, inspeccionar la piel y la mucosa bucal.

- Comprobar el color, cantidad y consistencia de las secreciones.

Tabla. No.3 Plan de Cuidados de Enfermería; Hipertermia

DIAGNÓSTICO DE ENFERMERÍA: Desequilibrio nutricional: ingesta inferior a las necesidades r/c incapacidad para ingerir alimentos (estado de sedación) $\mathrm{m} / \mathrm{p}$ pérdida de peso, palidez de mucosas DOMINIO: 02: nutrición

CLASE:01: ingestión

\section{RESULTADOS ESPERADOS (NOC)}

Estado nutricional
INDICADORES

- Ingestión de nutrientes

\section{ESCALA DE MEDICIÓN}

PUNTUACIÓN DIANA

11) Grave ........1

12) Sustancial...2

13) Moderado....3

14) Leve............

15) ninguno.....5
Inicial Esperada Final

3

3

$\begin{array}{lll}3 & 4 & 4\end{array}$

INTERVENCIÓN: Terapia nutricional

\section{ACTIVIDADES:}

- Administrar alimentación enteral, cuando sea preciso

INTERVENCIÓN: Alimentación enteral por sonda ACTIVIDADES:

- Observar si la colocación de la sonda es correcta inspeccionando la cavidad bucal.

- Elevar el cabecero de la cama de 30 a $35^{\circ}$ durante la alimentación 
Tabla 4. Plan de Cuidados de Enfermería exceso de volumen de líquidos

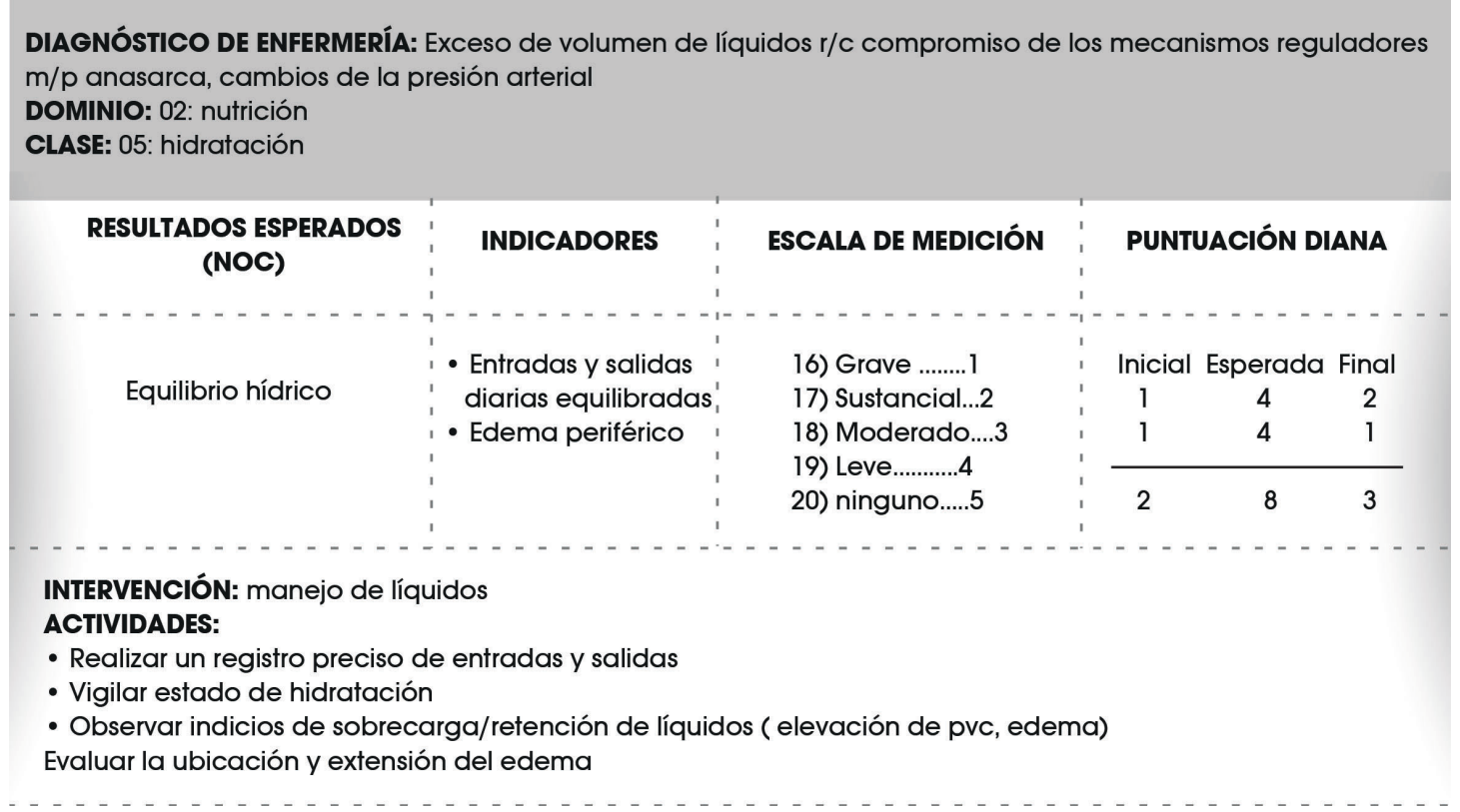

\section{Resultados}

Para el diagnostico de mayor prioridad (riesgo de perfusión tisular cerebral ineficaz), se consideraron los indicadores presión sanguínea diastólica,

Presión sanguínea sistólica, Deterioro cognitivo>>, se logró avanzar de una escala apaDiana inicial de 4, obteniendo una final de 9, logrando avanzar en un 90\% en la evaluación final.

Gráfica 1. Evaluación

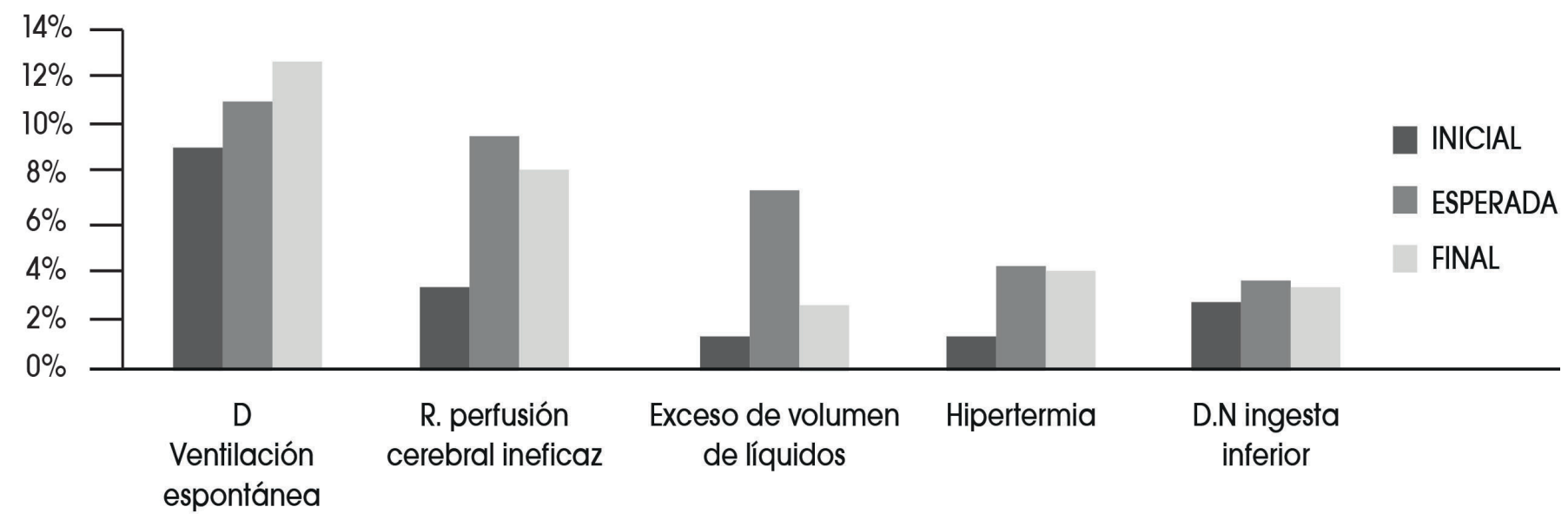




\section{DISCUSIÓN}

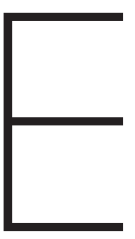

I realizar las intervenciones de enfermería de forma sistematizada a partir del proceso enfermero, se establece un método científico en donde cada intervención se fundamenta y da pauta a la aplicación de un cuidado especializado, dirigido hacia la mejora de la persona desde el punto de vista individual y con ello detectar oportunamente signos y síntomas de alarma, así como posibles complicaciones. Lo innovador en el presente artículo, es que se pretende eliminar la idea de que un diagnóstico de riesgo no puede ser uno de los prioritarios, cabe mencionar que la Taxonomía NANDA no cuenta con un diagnostico real que haga alusión a la presente patología ya que, las definiciones no se adecuan a la fisiopatología y los diagnósticos aplicados en publicaciones pasadas como "Intervenciones de enfermería en la persona con vaso espasmo cerebral secundario a hemorragia subaracnoidea aneurismática" en el

\section{REFERENCIAS BIBLIOGRÁFICAS}

1. Comision Permanente de Enfermeria. Manual de Plan de cuidados (Online):; 2011 (Citado 2016 Noviembre 30. Disponible en: http://www.salud. gob.mx/unidades/cie/cms_cpe/

2. NANDA International Inc. Diagnosticos Enfermeros Definiciones y Clasificación 2015-2017. 3 ed. H. TH, Shigemi K, editors. Madrid: El Sevier; 2015.

3. S. M, Johnson M, Maas ML, Swanson E. Clasificación de Resultados de Enfermería (NOC). 5 ed. Madrid: El Sevier; 2013

4. GM B, BHHK, McCloskey-DJ. Clasificacion de intervenciones de Enfermería (NIC). 6 ed. Madrid: El Sevier; 2013

5. Vallejo JCV. Sobre el modelo AREA y el proceso enfermero. Inquietudes 2006; 7 (35)

6. Sevilla R. Papel de enfremeria en el juicio clinico: la valoracion y el diagnóstico. Enfer Cardiol 2014; 1(61)

7. Brito PRB. Redes y diagnósticos enfermeros, en Rev Enfer 2008; 7(2)

8. Montiel LFH. Hemorragia subaracnoidea en el servicio de Admisión Continua del. Medicina Interna de México. 2010; 26(3) volumen 14 mayo 2015,12 hacen alusión a diagnósticos que ahora han sido eliminados de la nueva edición de la taxonomía. De igual manera se implementa el uso de la frasecomo lo demuestra para referirse a la evidencia del riesgo que existe y que, los planes de cuidado de enfermería no se hubiera detectado e implementando de manera oportuna la salud del usuario podría haberse visto comprometida.

\section{CONCLUSIÓN}

Implementar el modelo de análisis de resultados del estado actual nos ofrece las siguientes ventajas; identificación del diagnóstico principal, los diagnósticos secundarios con base al nivel de prioridad para su intervención, y diagnósticos de riesgo, de tal forma que se establecen los resultados. Las relaciones se han constituido con facilidad, teniendo en cuenta los criterios de resultados que van a medir la evolución del paciente.

9. Guyton CA HE. Tratado de Fisiología Médica. 11 ed. Madrid: El Sevier; 2006

10. Pedro Luis Rodríguez García DRG. Revista cubana de neurologia y neurocirugia. (En linea): 2017 (Citado 2016 noviembre 29. Disponibleen:file:///C: Users/usuario/Downloads/Dialnet-HemorragiaSubaracnoidea-4790507\%20 (3).pdf.

11. Hospital General de Mexico. Guias diagnosticas de Neurología y Neurocirugía. (En linea).: 2011 (Citado 2016 Noviembre 30. Disponible en: http://www.hgm.salud.gob.mx/descargas/pdf/area_medica/neuro/ guias/hemorragia_subaracnoidea.pdf.

12. Bravo Castrejón DA. Intervenciones de enfermería en la persona con vasoespasmo cerebral secundario a hemorragia subaracnoided aneurismática. Revista de Enfermería Neurológica. 2015; 14(1).

13. Suarez JI TRSW. Aneurysmal Subarachnoid Hemorrhage. N Engl J Med 2006:354:387-96.

14. Cruz MP. Enfermera; unidad de medicina interna. Inquietudes; 1999 ; 1 (41)

Enf Neurol Vol. 16. No. 3 septiembre - diciembre 2017 